This is a postprint version of the following published document: Sara Fernández; Ignacio de Miguel; Ramón J. Durán; Jesús Javier Castro; Noemí Merayo; Juan Carlos Aguado; Lidia Ruiz; Patricia Fernández; Rubén M. Lorenzo; Evaristo J. Abril. Technoeconomic comparison of dynamic traffic grooming strategies for elastic optical networks. In: 2017 19th International Conference on Transparent Optical Networks (ICTON). DOI: https://doi.org/10.1109/ICTON.2017.8025127

(C) 2017 IEEE. Personal use of this material is permitted. Permission from IEEE must be obtained for all other uses, in any current or future media, including reprinting/republishing this material for advertising or promotional purposes, creating new collective works, for resale or redistribution to servers or lists, or reuse of any copyrighted component of this work in other works. 


\title{
Techno-economic Comparison of Dynamic Traffic Grooming Strategies for Elastic Optical Networks
}

\author{
Sara Fernández, Ignacio de Miguel, Ramón J. Durán, Jesús Javier Castro, Noemí Merayo, \\ Juan Carlos Aguado, Lidia Ruiz, Patricia Fernández, Rubén M. Lorenzo, Evaristo J. Abril \\ Universidad de Valladolid, E.T.S.I. de Telecomunicación. Campus Miguel Delibes, 47011 Valladolid, Spain \\ e-mail:ignacio.miguel@tel.uva.es
}

\begin{abstract}
We extend past work presented at ICTON 2015, where we reviewed and extended a number of dynamic traffic grooming algorithms proposed by Zhang et al. for elastic optical networks. In that paper we showed that, on the one hand, different grooming policies lead to different connection blocking ratios and have different associated economic costs. On the other hand, different types of transceivers (supporting a single type of modulation format, or being able to generate different ones) also lead to different results in terms of those parameters: blocking ratio and cost. So, a question arises: what is the best option when designing an elastic optical network? Is it better to use a cost-effective grooming policy with transceivers supporting several modulations, or to use cheaper fixed-modulation transceivers combined with an effective policy in terms of blocking ratio? In this paper, we analyze which combination of transceiver type and grooming policy has advantages from a technoeconomic perspective (considering both CAPEX and OPEX), while providing a target performance in terms of blocking ratio.
\end{abstract}

Keywords: elastic optical networks, techno-economic analysis, dynamic traffic grooming, modulation formats.

\section{INTRODUCTION}

The exponential traffic growth is having great impact on the development of transport networks and, in particular, optical networks. Within this type of networks, elastic optical networks are receiving great attention [1]. These networks allow the establishment of optical connections (or lightpaths) with different spectral bandwidths, which leads to a more efficient use of resources, since the amount of optical spectrum assigned to a lightpath can be adapted to its needs and accommodate traffic variations. The modification of spectral bandwidth can be achieved by varying the number of subcarriers that are assigned to each lightpath, and can be complemented with the use of different modulation formats (with different spectral efficiencies). These features provide flexibility, but also increase the complexity of network control mechanisms.

This paper focuses on the problem of dynamic traffic grooming in elastic networks. Users can request connections at binary rates that differ from the capacity of a lightpath. Although the property of elasticity provides a solution to adapt the capacity of a lightpath to user demands, a more efficient use of the resources can be achieved if a technique called grooming is also used. That technique consists in aggregating several user connections on a single lightpath, and ensuring that traffic reaches the destination through a sequence of one or more lightpaths (either already existing or new ones). The resolution of the problem of dynamic traffic grooming therefore determines how to route the traffic demands that the network receives, whether through the electrical layer, through the optical layer or through a mixture of both, allocating resources as required.

Our starting point was the proposal by Zhang et al. [2]. They propose a method and set of policies for dynamic traffic grooming, and analyze their performance assuming that transceivers use the QPSK modulation format and an ideal physical medium. Specifically, they propose the following policies:

- Minimum Number of Lightpaths (MinLPs): this policy aims at minimizing the number of lightpaths newly established in the network (i.e., it reuses lightpaths at most as possible by grooming connections on them).

- Minimum Number of Virtual Hops (MinHops): this method aims at minimizing the number of lightpaths traversed by a connection.

- Minimum Number of Traffic Hops in the Physical Network (MinTHP): this policy aims at minimizing the number of fibers (physical links) that are traversed by the connection.

- Load-Balancing (LB): this policy aims at balancing the load carried by the fibers (with the final objective of minimizing the blocking probability).

These policies can be combined with a technique called Spectrum Reservation (SR), which pre-reserves spectral resources for lightpaths in order to facilitate potential bandwidth expansions in the future.

In ICTON 2015 [3], we presented an extension of that study. We analysed the network performance when other modulation formats (16QAM and 64QAM) are employed and when considering physical impairment issues. Moreover, we also analysed how the policies by Zhang et al. [2] can be used in the operation of a heterogeneous network where QPSK, 16QAM and 64QAM modulation formats coexist, and we also performed an initial techno-economic analysis. The techno-economic model was based on a proposal of the European CHRON project [4,5], and took into account the cost of transponders, optical amplifiers, optical cross-connects and IP/MPLS nodes, as well as the consumption of electrical power, rental, maintenance and repair costs 
(normalized, so that 1 cost unit, c.u., is the cost of one $10 \mathrm{G}$ fixed transponder). The techno-economic study showed that different traffic grooming policies lead to different connection blocking ratios and have different associated economic costs. Moreover, different types of transceivers (supporting a single type of modulation format, or being able to generate different ones) also lead to different results in terms of those parameters (blocking probability and cost). That observation triggered the question of determining which combination of transceiver type and grooming policy has advantages from a techno-economic perspective, while providing a target performance in terms of blocking ratio. This paper aims to answer that question.

\section{SETTING UP THE RESEARCH QUESTION: A QUICK REVIEW OF OUR ICTON 2015 RESULTS}

As reported in [3], we have programmed an elastic network simulator in the OMNeT++ simulation framework [6]. We have considered the NSFNet topology, which consists of 14 nodes, $2 \times 21$ unidirectional links and has a diameter of 3 hops, assuming that each link has a spectrum of $1 \mathrm{THz}$ divided into $5 \mathrm{GHz}$ slots. Each transponder is able to transmit employing a maximum bandwidth of $50 \mathrm{GHz}$, and guard bands of $10 \mathrm{GHz}$ are used. The supported modulation formats are QPSK, 16QAM and 64QAM, with bit rates per slot of 10, 20 and $30 \mathrm{~Gb} / \mathrm{s}$, respectively. All links of the network are assumed to be of the same length and are normalized to the optical reach of the 64QAM modulation format (i.e., the optical reach of 64QAM is one hop). The optical reach of the 16QAM and QPSK modulation formats are 4 hops and 16 hops, respectively (in order to be consistent with, e.g., [4]). Unidirectional connection requests are generated according to a Poisson process, and the duration of the connections are exponentially distributed. The source and destination nodes of the requests are selected using a uniform distribution, and the requests demand $10 \mathrm{~Gb} / \mathrm{s}, 40 \mathrm{~Gb} / \mathrm{s}$ or $100 \mathrm{~Gb} / \mathrm{s}$ with proportion 10:4:1 (as in [2]).

Although we studied different metrics, we focus on the blocking probability, BBR (Bit rate Blocking Ratio), calculated as the quotient between the sum of the bit rates associated with blocked requests and the sum of the bit rates associated with all the requests. Specifically, we analysed the relationship of BBR with the modulation format used, and with the traffic grooming policies proposed in [1], all these policies combined with the spectrum reservation (SR) technique previously mentioned (MinHops-SR, MinLPs-SR, MinTHP-SR, LB-SR).

Homogeneous and heterogeneous scenarios (where transponders can generate a single modulation format or several ones, respectively) were studied, considering both an ideal and a realistic physical medium. In all cases, the best results in terms of BBR were obtained when using LB-SR as the grooming policy [3]. It is worthy to note that when considering a realistic physical medium, the optical reach of 64QAM (which is only one hop) leads to very high BBR for all traffic loads, except when the MinTHP-SR or LB-SR policies are used (since these policies usually lead to establishing lightpaths of just one hop). For that reason, when using heterogeneous transponders, if 64QAM carriers are required to establish a connection, only MinTHP-SR or LB-SR policies are allowed (see [3] for details). Therefore, in the following figures, we will represent the policies as Policy-1 / Policy-2, where Policy-1 is the main traffic grooming policy employed in the study except when 64QAM subcarriers are involved, in those cases using Policy-2.

As shown in Fig. 1.a, when considering a realistic physical medium, the use of heterogeneous transponders leads to reductions in BBR when compared to the use of homogeneous transponders (with the same main grooming policy). This is due to the fact that the former option takes advantage of the spectral efficiency provided by the 64QAM modulation format when the distances are short, and the robustness of QPSK when the involved distances are high.

The operational expenditure (OPEX) results for that scenario are shown in Fig. 1.b. It is important to note that an initial dimensioning phase was not carried out in this study, that is, the simulation was launched with the conditions already commented and the number of resources used was analyzed. Based on this, we calculated the capital and operational costs, CAPEX and OPEX. Obviously, as the traffic load increases, the costs also increase as the number of active devices that are required to be able to carry that load also increases. Moreover, although the LB-SR grooming policy is the most effective in terms of BBR (Fig 1.a), it leads to higher costs (Fig. 1.b). By contrast, MinHops-SR offers the worst results of all the policies in terms of BBR, but leads to lower costs than other policies.

However, the comparison made so far must be taken with caution. The combination of heterogeneous transponders with the MinHops-SR policy seems (in general) the most economical option (Fig. 1.b), but it leads to a BBR higher than other alternatives for the same traffic load (Fig. 1.a). This is due to the fact that the network has not been previously dimensioned. Therefore, to make a fair comparison, it would be necessary to dimension the network so that the different combinations of transponder type and grooming policy give rise to the same BBR value for the same traffic load, and then compare the costs under those conditions.

Nevertheless, with this initial study we showed how different grooming policies lead to different BBR values, but also have different costs associated with them. On the other hand, different types of transponders also give rise to different values of $\mathrm{BBR}$ and cost. The next objective is therefore to answer this question: What combination of grooming policy and transponder is more economical while guaranteeing a certain BBR? Is it better to use a cost-effective grooming policy with heterogeneous transponders, or to use homogeneous (cheaper) transponders along with BBR-efficient grooming policies (but not cost-effective)? 

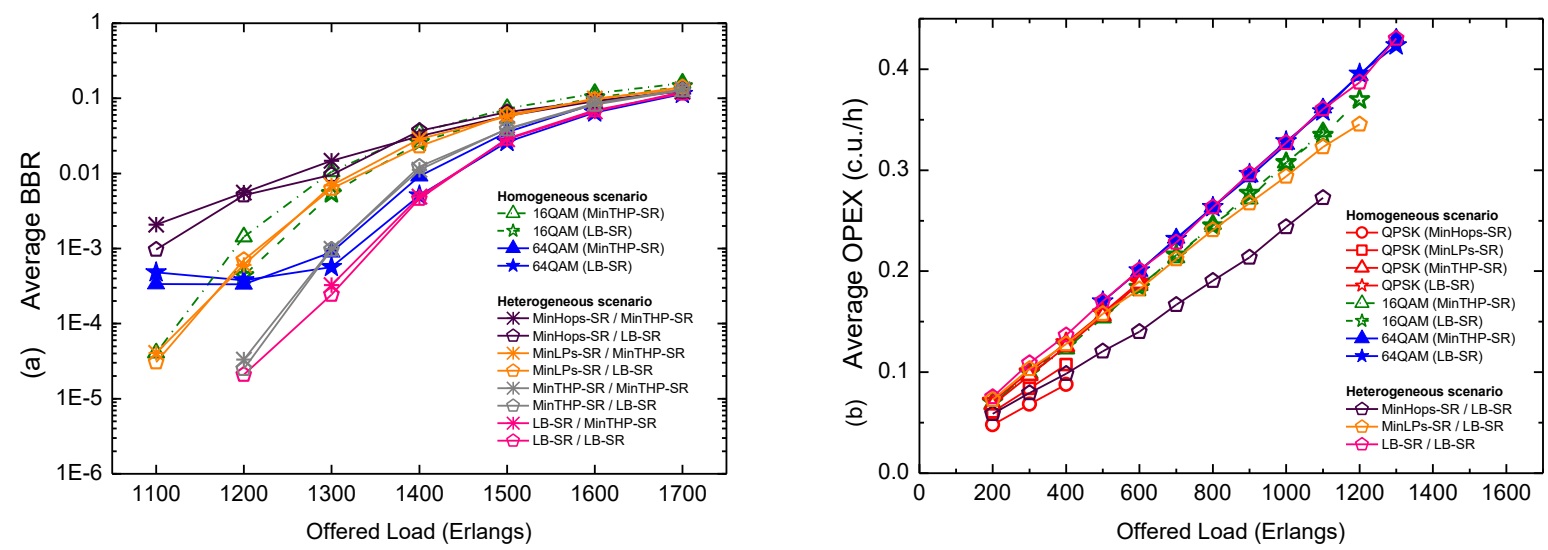

Figure 1: Reproduced from [3], (a) BBR for different policies in a heterogeneous scenario assuming a realistic physical medium. For comparison purposes the results for the most effective policies and modulation formats in terms of BBR for homogeneous scenarios are also shown (only high traffic loads are represented)

(b) OPEX for different policies assuming a realistic physical medium (all plotted results fulfill that BBR $<10^{-3}$ ).

\section{TECHNO-ECONOMIC COMPARISON}

In order to answer the question mentioned above, we have first applied a dimensioning procedure. For each traffic load, grooming policy and type of transponder, the network has been dimensioned to determine the set of resources necessary to obtain approximately $\mathrm{BBR} \approx 10^{-3}$. Anyway, it is important to note that the available spectrum is not varied during the dimensioning procedure, but only the number of components (transponders, wavelength selective switches and IP ports) in each node. By matching all the scenarios so that they provide the same BBR (approximately), a fair comparison of costs can be made. For the dimensioning procedure, the starting point is the simulation study leading to Fig. 1.a (considering also additional results for lower loads and for the QPSK modulation format). For each combination of transceiver type and grooming policy, the configuration of the network that provides $\mathrm{BBR} \approx 10^{-3}$ at a certain traffic load is taken as a reference (e.g., for heterogeneous transponders with MinHops-SR/LB-SR, the reference configuration is that obtained at 1100 Erlangs as shown in Fig. 1.a). Now, if we reduce the number of resources in each node (e.g., to $60 \%$ of the original number), the CAPEX and OPEX will also reduce, but the network will support a lower traffic load than in the original configuration if a $\mathrm{BBR} \approx 10^{-3}$ is still desired. Then, by means of simulation, we determine which traffic load leads to $\mathrm{BBR} \approx 10^{-3}$ for different scaling factors, and compute the CAPEX and OPEX associated to those network configurations.

Fig. 2.a shows the BBR obtained in a new set of simulations for different combinations of transceiver type and grooming policy when the network has been previously dimensioned as just explained. Due to the granularity of the scaling factor (steps of 10\%), and of the traffic loads tested (steps of 50 Erlangs), the BBR deviates from the target value of $10^{-3}$ in many cases (as shown in that figure). In order to assess the impact of these deviations, for the combination of QPSK modulation format with LB-SR policy, two sets of configurations have been considered, one with BBR slightly below $10^{-3}$, and the other above (Fig. 2.a). However, as shown in Fig. 2.b and 2.c, the CAPEX and OPEX are very similar in both cases, despite their differences in BBR.

In terms of costs, the results show that the lower CAPEX (Fig. 2.b) is obtained with the homogeneous QPSK scenario and LB-SR, although it is not able to support loads higher than 650 Erlangs (with BBR $\approx 10^{-3}$ ) due to spectrum limitations. That is, for higher loads at that scenario is the lack of spectral resources rather than the lack of components which leads to blocking events. From the point of view of OPEX (Fig. 2.c), the heterogeneous scenario with MinHops-SR/LB-SR has, in general, the lowest cost. It is therefore necessary to analyze whether the annual savings in OPEX will compensate for its higher CAPEX (compared to QPSK with LB-SR) in a reasonable period of time. However, for example, for a traffic load of 500 Erlangs, that would take about 20 years, which is a too large period of time taking into account the rate at which new technologies appear and are introduced in optical networks.

The completion of this analysis leads to the following conclusion for this case study concerning the NSFNet:

- If the expected loads are low (up to 650 Erlangs), the most economical viable solution is the use of QPSK homogeneous transponders and the LB-SR traffic grooming policy.

- If loads between 650 and 1100 Erlangs are expected, the best option is the use of heterogeneous transponders with the MinHops-SR/LB-SR policy.

- If loads between 1100 and 1300 Erlangs are expected the best option is the use of 16QAM homogeneous transceivers with the LB-SR policy.

Therefore, the expected traffic load on the network and its expected evolution are key issues in choosing the most appropriate type of transponder and grooming policy. 

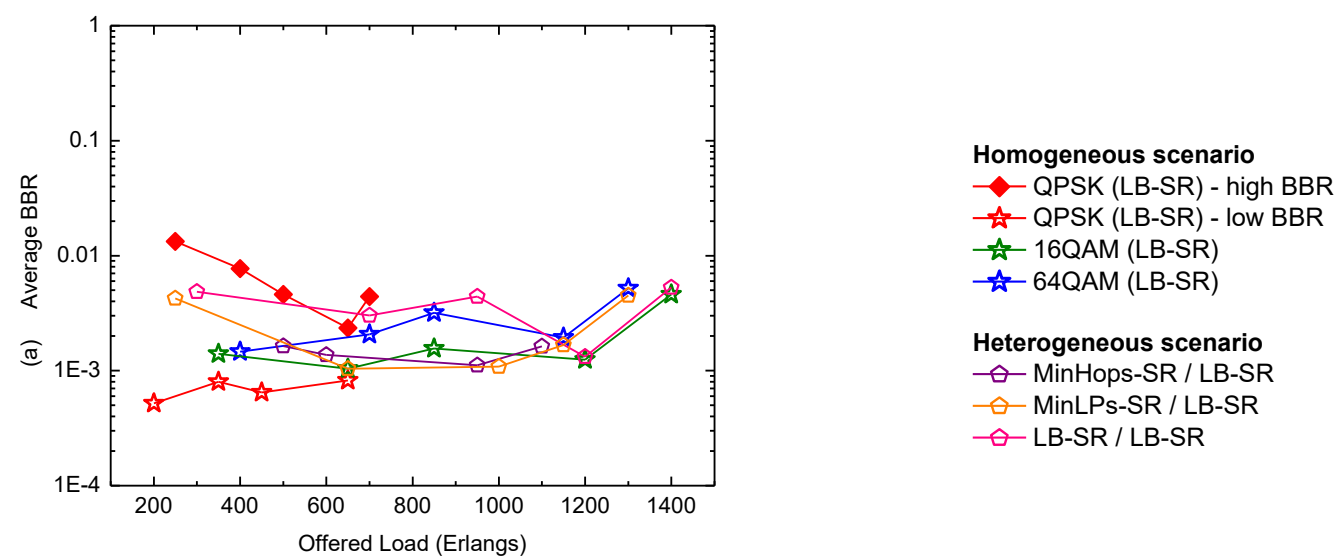

Heterogeneous scenario

A- MinHops-SR / LB-SR

- MinLPS-SR / LB-SR

- LB-SR / LB-SR
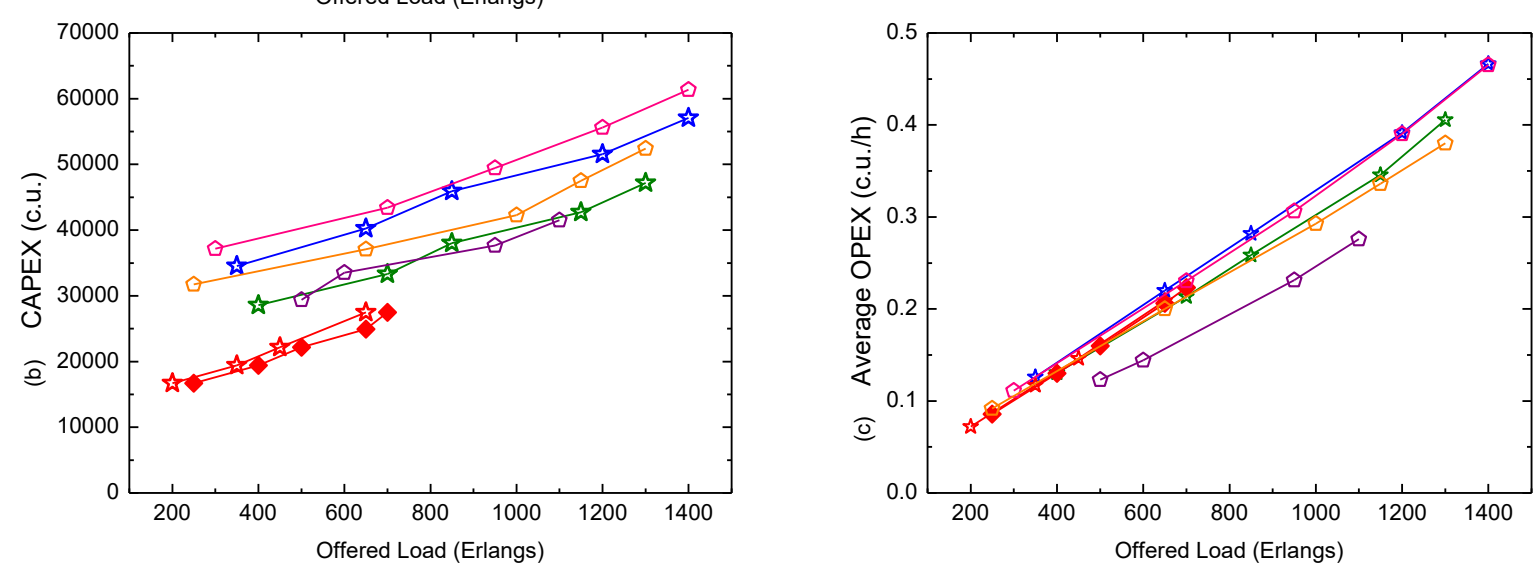

Figure 2: (a) BBR (b) CAPEX (c) OPEX for different policies assuming a realistic physical medium.

\section{CONCLUSIONS}

Different traffic grooming policies and different types of transponders in elastic optical networks give rise to different blocking ratios, but also have different economic costs associated with them. Therefore, the optimal configuration from a techno-economic point of view must be determined, which involves dimensioning and analysing the network to ensure similar performance among the different alternatives. A use case, related to the NSFNet topology, with a specific configuration, has been studied. In this case, we have shown that for low traffic loads the most viable solution is to use QPSK transponders together with an efficient grooming policy in terms of blocking probability: LB-SR. At medium loads the best option is to use heterogeneous transponders (more expensive than the previous ones) along with the MinHops-SR grooming policy, which is less efficient in blocking probability than LB-SR, but more cost-efficient. Finally, for high loads, the best option is to use 16QAM transponders together with the LB-SR policy.

\section{ACKNOWLEDGEMENTS}

This work has been supported by Consejería de Educación de la Junta de Castilla y León (Ref. VA353A11-2), and by Spanish Ministry of Economy and Competitiveness (TEC2014-53071-C3-2-P, TEC2015-71932-REDT).

\section{REFERENCES}

[1] I. Tomkos, et al:: A tutorial on the flexible optical networking paradigm: State of the art, trends, and research challenges, Proc. of the IEEE, vol. 102, pp. 1317-1337, Sep. 2014.

[2] S. Zhang, C. Martel, B. Mukherjee: Dynamic traffic grooming in elastic optical networks, IEEE J. Sel. Areas. Commun., vol. 31, pp. 4-12, Jan. 2013.

[3] J.J. Castro, S. Fernández, I. de Miguel, R.J. Durán, N. Fernández, N. Merayo, J.C. Aguado, P. Fernández, R.M. Lorenzo, E.J. Abril: A comparison of dynamic traffic grooming algorithms for elastic optical networks, in Proc. ICTON 2017, Budapest, Hungary, July 2015, paper Tu.B3.1.

[4] J.L. Vizcaino, et al.: Cost evaluation for flexible-grid optical networks, in Proc. Globecom Workshops 2012, pp. 358-363, Dec. 2012.

[5] E. Palkopoulou, et al.: Cognitive Heterogeneous Reconfigurable Optical Network: A techno-economic evaluation, in Proc. Future Network and Mobile Summit 2013, July 2013.

[6] OMNeT++, http://www.omnetpp.org. 